\title{
Indikationen und Methoden der konservativen Behandlung von Wirbelfrakturen
}

\author{
Erol Gercek, Holger Meinig, Frank Hartmann, Eric Hanke, Sven Oliver Dietz, \\ Pol Maria Rommens
}

\section{Zusammenfassung}

Bei der Behandlung von Wirbelfrakturen stellte die konservative Therapie unabhängig von der Verletzungsschwere lange Zeit den Goldstandard dar. Mit Einführung und Etablierung verschiedenster Operationsverfahren sank der Stellenwert der konservativen Therapie, was vor allem die Behandlung der instabilen Verletzungen betrifft. Nach wie vor wird die konservative Therapie in der Behandlung der stabilen Frakturen eingesetzt. Die Grenzbereiche liegen bei den sog. semistabilen Verletzungen. Valide Vergleiche von Ergebnissen nach konservativer und operativer Behandlung von Wirbelverletzungen liegen nicht vor, da prospektiv vergleichende Studien und die exakte Klassifikation der Verletzungen kaum gelingen. Die Definition von Instabilität und Stabilität stellt ein weiteres Hindernis dar, weil eine klare Unterscheidung, trotz vieler Versuche und der hohen Varianz der Verletzungstypen, nicht sicher erfolgen kann. Prospektiv untersuchten wir 70 stabile bis semistabile Wirbelfrakturen, die in 2 Gruppen randomisiert eingeteilt wurden. Es wurde dabei die sofortige frühfunktionelle Mobilisation mit 3-PunktKorsett gegen eine funktionelle Thera- pie mit initialer Immobilisation und Mobilisation nach 1 Woche mit 3Punkt-Korsett untersucht. Unterschiede ließen sich weder im klinischen noch im funktionellen Outcome finden. Verschiedene konservative Behandlungsansätze haben sich in den letzten 100 Jahren etabliert, wobei diese von der vollständigen Immobilisation über geschlossene Repositionsmanöver, Mieder- und Korsettversorgungen bis zur frühfunktionellen Therapie reichen. Gerade die frühfunktionelle Therapie gilt heute im deutschsprachigen Raum als anerkanntes konservatives Verfahren.

\section{Indications and Methods for the Conservative Treatment of Spine Fractures}

In the treatment of spine fractures regardless of the gravity of the injury conservative therapy has represented the gold standard for a long time. With the introduction and establishment of different operation procedures the value of the conservative therapy has declined, especially with regard to the treatment of unstable injuries. Conservative therapy still has its place in the treatment of the stable fractures. The borderline areas are occupied by the so-called semi-stable injuries. Valid comparisons of results after conservative and surgical treatment of spine injuries are not available because prospective comparative studies and the exact classification of the injuries are hardly ever successfully reported. The definitions of instability and stability provide another obstacle because a clear differentiation, in spite of many attempts and the high variance of the injury types, cannot be given with certainty. We have prospectively examined 70 patients with stable to semistable spine fractures who were randomised into 2 groups. Immediate earlyfunctional mobilisation with 3-point corsets was examined in comparison to functional therapy with initial immobilisation and mobilisation with 3point corsets after 1 week. No differences could be found either in the clinical or in the functional outcome. Various conservative treatment modalities have set up during the last 100 years and range from entire immobilisation about a closed repositioning manoeuvre, corsage and corset care up to the early-functional therapy. Nowadays only the early-functional therapy is valid as an approved conservative procedure in the German-speaking countries.

\section{Einleitung}

Wirbelsäulenverletzungen, sei es ossärer oder nicht ossärer Art, mit und ohne assoziierte neurologische Schädigungen führen, verglichen mit den anderen Organsystemen des menschlichen Körpers, zu den schlechtesten funktionellen Ergebnissen und der höchsten Rate an

OP-JOURNAL 2009; 25: 4-10

(C) Georg Thieme Verlag KG Stuttgart · New York DOI 10.1055/s-0029-1185593
Langzeitschäden [1]. Die konservative Therapie war lange Zeit die einzige Therapieoption und gilt bis heute als Standardtherapie bei den weniger schweren Verletzungen. Voraussetzung für die Einschätzung der Verletzungsschwere ist das Verständnis vom mechanischen und neurologischen Gefährdungspotenzial, das aus den unfallbedingten morphologischen Veränderungen resultiert. In Abhängigkeit von der Verletzungsschwere wird eine geeignete Therapie gewählt, die heute in erster Linie durch radiologische und neurologische Erkenntnisse be- stimmt wird. Das Therapieziel bleibt nach wie vor die Wiederherstellung einer schmerzfreien statischen, dynamischen und protektiven Funktion der Wirbelsäule.

Drei verschiedene konservative Behandlungsansätze für die häufigen Kompressionsfrakturen mit Keilwirbelbildung im Bereich Th12-L3 werden postuliert [2]:

1. Geschlossene Reposition und Ruhigstellung im Gipsmieder [3].

2. Bettruhe ohne Reposition, gefolgt von späterer funktioneller Therapie [4]. 

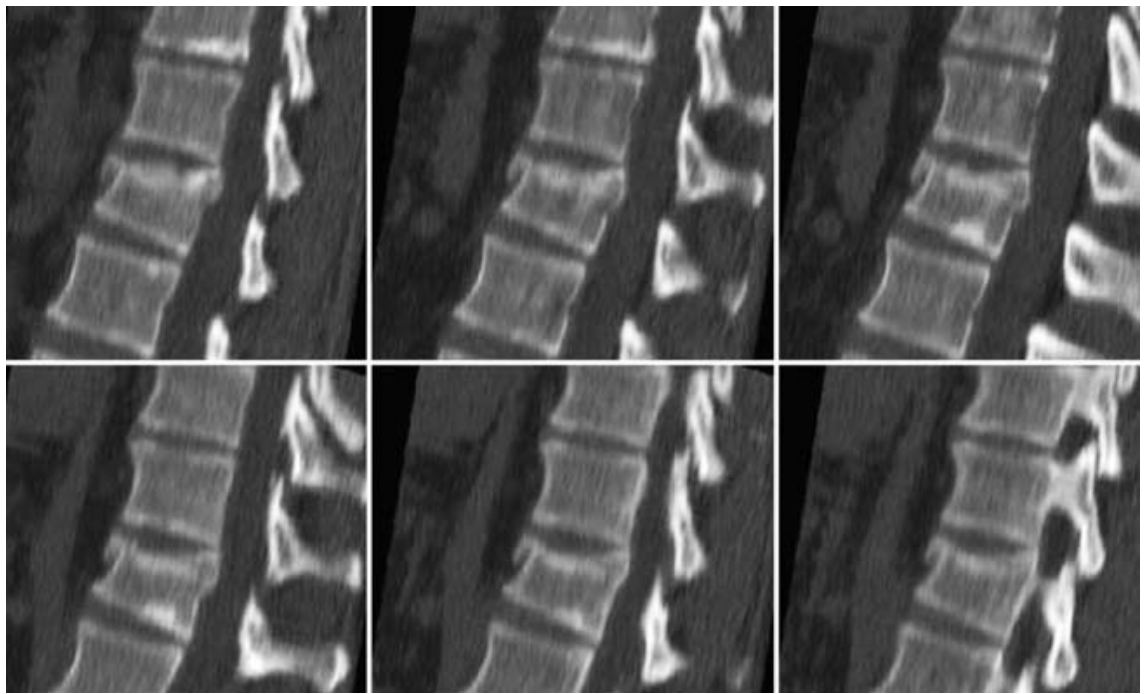

Abb. 1 CT-Darstellung einer kranialen Berstungsfraktur von LWK 1.

3. Primäre funktionelle Therapie ohne Reposition mit rascher Mobilisierung, wobei, gemäß den Ergebnissen dieser Untersuchung, eine initiale Immobilisation kein besseres Endergebnis erzeugt.

Die bis Mitte der 80er-Jahre des letzten Jahrhunderts überwiegend durchgeführten konservativen Therapien wurden in den letzten 25 bis 30 Jahren vor allem bei den sog. instabilen Verletzungen verlassen, hin zu operativen Verfahren. In dieser Arbeit wird die konservative Therapie mit all ihren Facetten beleuchtet und diskutiert sowie die Ergebnisse einer eigenen prospektiven Untersuchung zur Frühmobilisation im Rahmen der konservativen Therapie dargestellt.

\section{Historischer Überblick}

Verletzungen der Wirbelsäule werden bereits seit Jahrtausenden immer wieder beschrieben. Exakte Beschreibungen von Verletzungen der Wirbelsäule finden sich von der Zeit des Homo primigenius bis in die Neuzeit. Die vermutlich älteste Beschreibung einer Halswirbelsäulenfraktur mit gleichzeitiger Lähmung stammt aus Ägypten (Papyrus Smith, ca. 1550 v. Chr.).

Erste Berichte über die Behandlung von Wirbelsäulenerkrankungen stammen von Hippokrates (460-377 v. Chr.). Er beschreibt detailliert die Behandlung von Skoliosen durch verschiedene Methoden der Extension. Hippokrates gilt noch heute als Inaugurator der allgemeinen akzeptierten Techniken von Redression und Reposition im Bereich der Wirbel- säule. Aus der berühmten Bank des Hippokrates, dem Scamnum entwickelten sich bis heute die allgemein verwendeten Extensionstische. Bei Wirbelfrakturen mit Lähmungen postulierte Hippokrates eine aussichtslose Situation ohne eine therapeutische Interventionsmöglichkeit.

Ende des 19. Jahrhunderts entwickelte der deutsch-russische Arzt Karl Rauchfuss (1835-1915) eine Vorrichtung aus Gurten, die die Entlastung erkrankter/ verletzter Abschnitte der Wirbelsäule (des liegenden Patienten) gestattet. Dabei wird eine Schwebelagerung des Körperstamms durch Vertikalzug mit einem breiten Gurt durchgeführt. Ursprünglich fand diese Technik Anwendung bei der Wirbelsäulentuberkulose, diente dann aber vor allem zur Aufrichtung bei Wirbelkörperkompressionsfrakturen im Lendenbereich, auch hier lagen die Ideen von Hippokrates zugrunde.

Eine neue Dynamik begann mit Entdeckung der Röntgentechnik im Jahr 1895 durch den Physiker Wilhelm Conrad Röntgen (1845-1923).

\section{Klassifikationen von Verletzungen der thorakolumbalen Wirbelsäule}

Frakturklassifikationen gelten als notwendige Werkzeuge zur Beurteilung des Schweregrads einer Verletzung und dienen als konzeptionelle Grundlage der Behandlung. Das Klassifikationswerkzeug sollte eine Behandlungsmethode nicht nur vorschlagen, es sollte dem Chirurgen auch eine recht exakte Schätzung des Outcomes dieser Behandlung ermögli- chen. Die Klassifikation von Brüchen der thorakolumbalen Wirbelsäule wird kontrovers diskutiert. Böhler machte die ersten Versuche einer Klassifikation 1929. Seit damals ist die Schwierigkeit einer kompletten Klassifikation von allen Autoren erkannt worden, die versuchen, ein System zu erstellen. Die Hauptschwierigkeit liegt in der Tatsache, dass, anders als bei Brüchen der langen Knochen, Wirbelbrüche durch Progression eine zunehmende Deformität entwickeln können und dass Wirbelfrakturen komplexe Verletzungen einer Struktur darstellen, die z.T. aus knöchernen Strukturen, z.T. aus Gelenken und Bändern besteht.

Das wachsende Aufkommen der Computertomografie (CT) in der 2. Hälfte der 70er-Jahre erlaubte einen neuen Einblick in die genaue Struktur von Wirbelverletzungen (Abb. 1). Die Möglichkeit der Abbildung der Wirbelsäule im Querschnitt lenkte die Aufmerksamkeit auf die Details der Brüche und die Kompromittierung des Spinalkanals, die nicht mit den traditionellen Belichtungstechniken erfasst wurden. Diese CT-Entdeckungen hatten eine Auswirkung auf die Entwicklung aktueller Klassifizierungssysteme, basierend auf der genaueren Beschreibung des Umfangs und des Ortes einer Verletzung.

Bemühungen in dieser Richtung in den frühen 80er-Jahren kumulierten im 3Säulen-Konzept von Denis [5], das das dominierende Klassifizierungssystem wurde. Die vordere Säule wird vom vorderen Längsligament, vom vorderen Annulus fibrosus und vom vorderen Teil des vertebralen Korpus gebildet; die mittlere Säule durch das hintere Längsligament, den hinteren Anulus fibrosus und die hintere Wand des vertebralen Korpus und schließlich die hintere Säule durch den hinteren knöchernen Komplex zusammen mit dem hinteren Ligamentkomplex.

Zwei neuere Bemühungen, die Klassifikationsentwürfe der thorakolumbalen Wirbelsäulenverletzungen $\mathrm{zu}$ rationalisieren, kamen 1994 auf. Es handelt sich einerseits um die Load-Sharing-Klassifikation, die von McCormak vorgeschlagen wurde [6]. Dieser Einteilung liegt eine spezifische Ausarbeitung des 3-Säulen-Systems von Denis zugrunde, wobei der Grad der Zerstörung des vertebralen Korpus zusammen mit der Lage der einzelnen Fragmente und dem Grad der Deformität beurteilt wird. Andererseits hat 
sich in den letzten Jahren im deutschsprachigen Raum zur Einteilung der Frakturen der Rumpfwirbelsäule die Klassifikation nach Magerl durchgesetzt [7]. Dieser Entwurf basiert auf den pathomorphologischen Eigenschaften der Verletzungen. Drei Hauptkategorien mit einem allgemeinen Verletzungsmuster, Typ und Form der Frakturen bilden diese Klassifikation (Abb. 2).

- Typ A: vertebrale Korpus-Kompression

- Typ B: vordere und hintere Elementverletzung mit Distraktion

- Typ C: vordere und hintere Elementverletzung mit Rotation

Es ist bemerkenswert, dass die Autoren das 3-Säulen-Konzept verließen und zum 2-Säulen-Konzept zurückkehrten. Sie gingen auch zurück zum mechanistischen Klassifikationsansatz mit Kennzeichnung der einzelnen Typen der Frakturen, so steht Typ A für Verletzungen durch Kompressionskräfte, Typ B für Distraktionskräfte und Typ C für Drehkräfte. Für die weitere Subklassifikation verwendeten die Autoren das allgemeine, von der Arbeitsgemeinschaft für Osteosynthesefragen (AO) entwickelte, 3-3-3 Rasterfeld. Subklassifikationen von TypB- und -C-Verletzungen folgen im Wesentlichen der Subklassifikation von Typ A. Diese Klassifikation suggeriert, dass jede Verletzung einer bestimmten Kategorie zugeordnet werden kann. Problematisch ist es jedoch, anhand der konventionellen Röntgenaufnahmen und der CT nicht ossäre Verletzungen sicher einzuordnen. So kann bei einer FlexionsDistraktionsverletzung mit Zerreißung der dorsalen Ligamente bei der Frakturdiagnostik mittels konventioneller Röntgenaufnahmen und CT anhand einer erweiterten Dornfortsatzdistanz eine BVerletzung vermutet, aber nicht bewiesen werden. Liegt bei diesem Fall gleichzeitig eine Kompressionsfraktur der vorderen Säule vor, kann die Läsion als A-Typ zunächst fälschlich eingeordnet werden. Techniken mit Magnetresonanztomografie (MRT) können diese wichtige Frage klären und können die Reproduzierbarkeit der Klassifikation erhöhen. Mit MRT kann in vielen Fällen auch präoperativ eine Aussage über die dorsalen Ligamente gemacht werden und somit eine genauere Klassifizierung erfolgen. Inwiefern die durch die MRT gewonnenen Zusatzinformationen die Wahl der Therapie beeinflussen, bedarf aber weiterer Abklärungen.

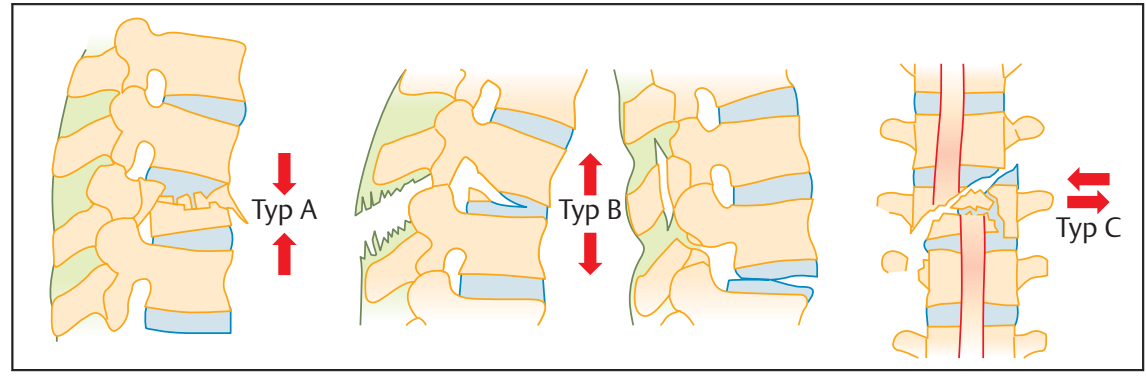

Abb. 2 Schema der Klassifikation nach Magerl, adaptiert von der AO.

Indikationen unter dem Aspekt

Stabilität - Instabilität

Stabilität und Instabilität können auf verschiedene Weisen definiert werden. Unterschieden wird zwischen morphologischen, funktionell anatomischen, neurologischen, biomechanischen und klinischen Beschreibungen. Allen Ansätzen der Stabilitätsdefinition ist gemeinsam, dass die Grenze zwischen einem stabilen und einem instabilen Zustand fließend ist und häufig nicht genau definiert werden kann. Durchgesetzt hat sich in den letzten Jahren die klinische Definition.

Zum Verständnis der mechanischen Stabilität gilt es, zunächst die Biomechanik des Wirbelkörpers und die Biomechanik des Bandapparats der Wirbelsäule zu verstehen. Während der morphologische Aufbau eines Wirbelkörpers vom 3. Halswirbel bis zum 5 . Lendenwirbel nur leicht variiert, nehmen Größe und Masse nach kaudal kontinuierlich zu. Man spricht hierbei von einer mechanischen Adaptation auf die zunehmende Kompressionslast der Wirbelkörper. Hinzu kommt ein Anstieg der Spongiosamasse, die zu einer deutlich höheren Stabilität der Wirbelkörper nach kaudal führt und somit den zunehmenden Lasten gerecht wird. Die zunehmende Frakturhäufigkeit im Alter beruht auf dem verminderten Mineralsalzgehalt des Knochens. So konnte gezeigt werden, dass ein Verlust von 25\% Knochensubstanz zu einer 50\%igen Stabilitätseinbuße bei isoliert axialer Belastung führt. Bei manifester Osteoporose kommt es zu Verlust der horizontalen Spongiosatrabekel, was zu einer mechanischen Schwächung der Vertikaltrabekel führt und die signifikante Stabilitätseinbuße der osteoporotischen Wirbelsäule erklärt. Dagegen ist die Kortikalis eines Wirbelkörpers, als zweite Struktur der axialen Kraftübertragung neben der Spongiosa, nur zu ca. 10\% an der axialen Kraftübertragung beteiligt [8].

Betrachtet man hingehen die Spongiosa eines durch Kompression frakturierten
Wirbels nach Ausheilung in Fehlstellung, so kann dieser Wirbelkörper in 50\% der Fälle sogar eine höhere Last tragen, ohne erneut zu brechen. Hier muss jedoch das Ausmaß der Höhenminderung des Wirbelkörpers berücksichtigt werden, von dem in hohem Maße die Stabilität abhängt. Sind bei Frakturen die Wirbelkörperendplatten beteiligt, so findet man eine enge Beziehung zum Degenerationsgrad der Bandscheiben. Bei nicht degenerierter Bandscheibe wirkt die komplette Kompressionskraft auf den Nucleus pulposus, was zu einer zentralen Krafteinwirkung auf die Endplatte führt. Bei Kompressionsverletzungen mit degenerativ veränderter Bandscheibe wird die axiale Kraft überwiegend über den Anulus fibrosus übertragen, wodurch häufiger eine Fraktur in den peripheren Endplattenbereichen auftreten. Die Gelenkfortsätze können je nach Stellung der Wirbelsäule bis zu 33\% der Kompressionslast und 45\% der Torsionskraft aufnehmen. Außerdem spielen sie eine wichtige Rolle für die Stabilität der Wirbelsäule, besonders bei Bewegungen in der Sagittalebene [8].

Von einem klinisch stabilen Zustand an der Wirbelsäule spricht man, wenn unter physiologischen Belastungen ohne Auftreten von Dislokationen ein $\mathrm{Zu}$ stand vorliegt, der ohne vernichtenden Schmerz, ohne neurologisches Defizit und ohne größere morphologische Veränderung einher geht [8]. Entsprechend wird von White die klinische Instabilität wie folgt definiert: „Clinical instability is the loss of ability of the spine under physiological loads to maintain its pattern of displacement so that there is no initial or additional neurological deficit, no major deformity and no incapacitating pain." Das Bewegungssegment als kleinste funktionelle Einheit spielt dabei die größte Rolle, deshalb wird meist von segmentalen Stabilitäten oder Instabilitäten gesprochen. 1963 versuchte Holdsworth, das Problem Stabilitätsbeurteilung mit einem säulenartigen Konzept zu lösen [9]. 
Er beurteilte die vertebrale Stabilität mit einem architektonischen 2-Säulen-Konzept. Diese Abstraktion ist bis heute im traumatologischen Bereich von wesentlicher Bedeutung. Die vordere Säule besteht aus den vertebralen Körpern und der intervertebralen Bandscheibe, die für ihn eine Synarthrose darstellt, die in Bezug auf ihre Stabilität auf den starken Anulus fibrosus angewiesen ist. Er nennt die hintere Säule „posterior ligament complex“. Dieser Komplex besteht aus den diarthrodialen apophysealen Gelenken (Facettengelenke), die durch die Kapsel, durch die intraspinösen Ligamente, supraspinösen Ligamente und das Ligamentum flavum stabilisiert werden. Holdsworths Theorie der Stabilität wird bestimmt durch die Integrität der hinteren Säule. Er nannte alle Verletzungsmuster mit einer intakten hinteren Säule stabil.

Die Weiterentwicklung stellte das 3-Säulen-Konzept von Denis dar [5]. Denis unterscheidet dabei erstmals Schweregrade der Instabilität:

- Die Instabilität 1. Grades ist eine mechanische Instabilität mit Gefahr der progressiven Kyphose. Sie tritt auf bei Kompressionsbrüchen mit hinterer Säulenverletzung, sowie bei Sicherheitsgurtverletzungen.

- Die Instabilität 2. Grades ist eine neurologische Instabilität. Sie kann verursacht werden durch Berstungsbrüche, bei denen in der frühen posttraumatischen Phase eine vertikale Einsinterung des verletzten Wirbels zu einer Verschiebung (Retropulsion) von Knochenfragmenten in den Spinalkanal führen kann. Damit ist die Gefahr einer posttraumatischen spinalen Stenose erhöht.

- Die Instabilität 3. Grades ist die Kombination einer mechanischen und neurologischen Instabilität. Frakturen mit ausgeprägten Dislokationen und komplexe Berstungsbrüche führen zu dieser Kategorie der Instabilität.

Eine Stabilität biomechanisch zu messen und zu definieren ist komplex. In-vitroUntersuchungen sind ungenauer als Invivo-Untersuchungen [10], weil die Simulation von Muskelkräften nicht möglich ist. Eine Einschätzung von Stabilität oder Instabilität bei einer akuten Verletzung vorzunehmen ist ebenfalls komplex, da Kenntnisse über die Grenzen vom stabilen zum instabilen Zustand fehlen. In der Regel orientiert man sich an den morphologischen Veränderungen, welche bei der bildgebenden Diag-

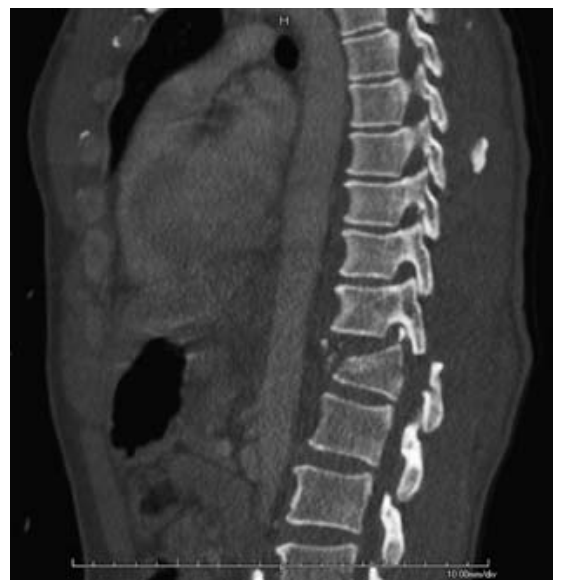

Abb. 3 Instabile Flexions-Distraktionsverletzung im Bereich des thorakolumbalen Übergangs.

nostik zutage treten. Diese morphologischen Veränderungen sind aber nur eine statische Momentaufnahme in einer vorgegebenen Haltung der Wirbelsäule, deshalb ist eine Stabilitätsbeurteilung anhand der Morphologie nur eingeschränkt möglich.

Orientierend an der Magerl-Klassifikation teilte auch Blauth Verletzungen in 3 Gruppen ein, er unterscheidet stabile, leichtgradig instabile und hochgradig instabile Situationen [11]. Kompressionsverletzungen der Typen A1 und A2 gelten als stabil, hier liegt meist keine Verletzung im Bereich der Bandscheibenräume vor. Eine größere Änderung der Frakturstellung ist weder in Ruhe noch bei Belastung zu erwarten. Ab Typ A2.3 (Kneifzangenbruch) wird eine leichtgradige Instabilität postuliert. Zu diesen gehören auch Berstungsfrakturen Typ A3, bei denen es sich um Kompressionsverletzungen mit Beteiligung der Hinterwand des Wirbelkörpers handelt.

Definitionsgemäß ist bei den Typ-A3Frakturen immer mindestens eine benachbarte Bandscheibe involviert, sodass auch von einer intervertebralen Instabilität gesprochen wird. Verletzungen von Ligamenten und Bandscheiben heilen mit Narbenbildung aus, die Funktion des Narbengewebes ist dabei nicht mehr mit der ursprünglichen Funktionsfähigkeit der Strukturen zu vergleichen. Diese Veränderungen führen somit zwangsläufig zu einer Stabilitätseinbuße. Es gibt bei Typ-A3-Frakturen jedoch auch Diskrepanzen in der Stabilitätsbeurteilung. Während die einen von einer Stabilität ausgehen [12], sprechen andere von einer Instabilität [13]. Bei den leichtgradigen Instabilitäten wird kein Risiko einer sekundären neurologischen Läsion gesehen, auch wenn eine funktionelle Therapie durchgeführt wird [14]. Es handelt sich um eine hochgradige Instabilität (Abb. 3), wenn beide Säulen betroffen sind, d.h. wenn eine Distraktionskomponente (Typ B) und/oder Rotationskomponente (Typ C) vorliegt.

Unabhängig von der Einteilung in TypA1-,-A2- oder-A3-Fraktur muss bei einer ausgeprägten kyphotischen Fehlstellung oder keilförmigen Deformation eines Wirbelkörpers mit einer segmentalen Überbelastung im Bereich Facettengelenke gerechnet werden. Diese Form der biomechanischen Instabilität kann auch zu einer seitenbetonten Kompression mit resultierenden skoliotischen Fehlstellungen führen, man spricht dann von einem asymmetrischen Verletzungsmuster.

Durch die Vielzahl unterschiedlicher Verletzungsmuster mit Übergangsformen ist eine differente Interpretation der Frakturen bezüglich der Stabilität/Instabilität möglich, die zu Verwirrungen und Kontroversen in der Indikation zur konservativen versus operativen Therapie führt. Blauth et al. zeigten bei isolierter Beurteilung der Primär-Röntgendiagnostik große Differenzen in der Zuverlässigkeit zwischen den Beobachtern. [15]. Zuallerletzt muss bei der Instabilität auch noch zwischen einer temporären und dauernden Instabilität unterschieden werden.

\section{Methodik}

Prospektiv erfassten wir die Daten und radiologischen Bildbefunde von 70 stabilen Frakturen der Brust- und Lendenwirbelsäule ohne neurologisches Defizit bei 51 Patienten im Zeitraum von 5/05 bis 5/07. Die Geschlechtsverteilung lag bei w:m 26:25, das Durchschnittsalter lag bei $44 \mathrm{LJ}$ (Spannweite 20.-65. Lebensjahr). Eingeschlossen wurden Patienten mit stabilen und semistabilen traumatischen Wirbelfrakturen der Brust- und Lendenwirbelsäule Typ A1 bis A3.1 (ausgenommen A2.3), wobei ein segmentaler Grund-Deckplatten-Winkel von nicht mehr als $15^{\circ}$ vorlag. Pathologische und osteoporotische Frakturen galten als Ausschlusskriterien, ebenso wie ein Alter über 65 Jahren, da hier zumeist eine gewisse osteopene Situation angenommen wurde. Alle Patienten, die eingeschlossen wurden, zeigten im CT lediglich eine Verletzung der vorderen und mittleren Säule im 3-Säulen-Modell. Die dorsale Säule war in keinem Fall betroffen, da 


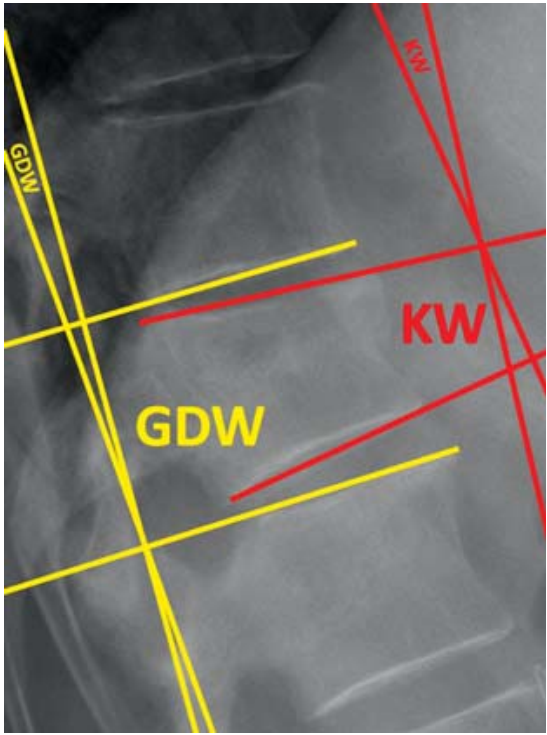

Abb. 4 Messmethode KW und GDW nach Daniaux direkt nach Trauma.

eine Beteiligung der dorsalen Säule ein hartes Zeichen für das Vorliegen einer Instabilität darstellt.

In dieser Untersuchung wurden 2 Verfahren zur konservativen Behandlung angewendet, die beide auf den Empfehlungen von Magnus beruhen [16]. In der 1. Gruppe erfolgte eine frühzeitige Mobilisation mit physiotherapeutischer Unterstützung ab 1. posttraumatischen Tag im 3-Punkt-Stützkorsett unter Gabe von Analgetika und keine Immobilisation mit Bettruhe. In der Gruppe 2 wurde eine konsequente vollständige Immobilisation mit Bettruhe bei flacher Lagerung für 1 Woche durchgeführt, wobei lediglich ein Drehen en bloc erlaubt war. Nach dieser Phase der Immobilisation erfolgte die Mobilisation mit physiotherapeutischer Unterstützung zunächst im Bewegungsbad unter Abnahme der Schwere schmerzabhängig für wenige Tage, gefolgt von einer Mobilisation im 3-PunktStützkorsett. Nach der ersten Mobilisation in Stand und Gang folgte in beiden Gruppen eine Röntgenkontrolle im Stehen in 2 Ebenen. Die Patienten wurden in der Regel einige Tage nach Mobilisation ins häusliche Umfeld entlassen, unter kontrollierter oraler Medikation des Schmerzes. Voraussetzung war eine sichere Mobilisation, die für die Aktivitäten des täglichen Lebens in häuslicher Umgebung ausreichend waren. In beiden Gruppen wurde das 3-Punkt-Stützkorsett für 6 bis 8 Wochen belassen.

Röntgenbilder vom Unfallzeitpunkt, vom Zeitpunkt nach der ersten Mobilisation (Aufnahme im Stehen) und vom
Tab. 1 Veränderung des Körperwinkels $(\mathrm{KW})$.

\begin{tabular}{lll} 
& $\begin{array}{l}\text { KW }\left[{ }^{\circ}\right] \\
\text { posttrauma }\end{array}$ & $\begin{array}{l}\text { KW }\left[{ }^{\circ}\right] \\
\text { Follow-up }\end{array}$ \\
\hline Gruppe 1 & $\varnothing-5,2$ & $\varnothing-7,0$ \\
\hline Gruppe 2 & $\varnothing-5,8$ & $\varnothing-7,2$
\end{tabular}

Zeitpunkt der letzten Follow-up-Evaluation wurden ausgewertet. Zur radiologischen Auswertung in den seitlichen Röntgenaufnahmen verwendeten wir die Messmethode nach Daniaux von 1986. Dabei wird vom frakturierten Wirbel der Winkel zwischen Grund- und Deckplatte $(\mathrm{KW}=$ Körperwinkel) bestimmt (Abb.4). Am KW kann man das Ausmaß der Wirbelkörpersinterung nach Fraktur erfassen. Als 2. Winkel wird der Winkel zwischen der Grundplatte des angrenzenden oberen Wirbelkörpers und der Deckplatte des benachbarten unteren Wirbels (GDW $=$ Grund-Deckplatten-Winkel) ausgemessen. Der GDW erfasst über die sagittale Deformierung des Wirbelkörpers hinaus Höhenminderungen der angrenzenden oberen und unteren Bandscheiben, die vor allem bei Berstungsbrüchen durch Eindringen der verletzten Bandscheibe in den frakturierten Wirbelkörper entstehen. Der KW und der GDW wurden bei einer Lordose mit einem positiven und bei Kyphose mit einem negativen Vorzeichen versehen. In der a.-p.-Aufnahme wurde der Skoliosewinkel nach Cobb bestimmt.

Die klinische Follow-up-Evaluation wurde mit dem Oswestry Score in Fragebogenform und der visuellen Analogskala (VAS) erfasst. Posttraumatisch, nach Erstmobilisation und zum Follow-up erfolgte die Erfassung der subjektiven Schmerzintensität mittels der visuellen Analogskala (VAS) (Skala von $0=$ kein Schmerz bis $10=$ maximal vorstellbarer Schmerz).

\section{Ergebnisse}

Die Auswertung der konventionellen Röntgenaufnahmen zeigte bei den meisten Patienten zum Unfallzeitpunkt das Vorliegen einer traumatisch bedingten segmentalen Kyphose. Der Körperwinkel zeigte in beiden Gruppen eine diskret zunehmende Kyphosierung (Tab. 1) der betroffenen Wirbelkörper, auch als Nachsinterung bezeichnet. Auch der GDW zeigte in beiden Gruppen nur eine diskret zunehmende Kyphosierung (Tab. 2), welche ebenfalls zwischen den Gruppen keine signifikanten Differenzen aufwies (Abb.5 und 6). Das Ausmaß der GDW-
Tab. 2 Veränderung des Grund-Deckplatten-Winkels (GDW).

\begin{tabular}{lll} 
& $\begin{array}{l}\text { GDW }\left[^{\circ} \text { ] }\right. \\
\text { posttrauma }\end{array}$ & $\begin{array}{l}\text { GDW }\left[^{\circ} \text { ] }\right. \\
\text { Follow-up }\end{array}$ \\
\hline Gruppe 1 & $\varnothing 0,2$ & $\varnothing-1,4$ \\
\hline Gruppe 2 & $\varnothing 1,4$ & $\varnothing 0,85$
\end{tabular}

Veränderung ist etwas geringer als das Ausmaß der KW-Änderung, was auf eine geringe Anzahl an Bandscheibenschäden durch die Verletzung bei den stabilen und semistabilen Frakturen zurückgeführt werden kann.

Die Auswertung des Oswestry-Scores ergab für Gruppe I einen Punkte-Score von durchschnittlich 20, für Gruppe II von durchschnittlich 18. Bis zum Zeitpunkt des letzten Follow-ups verbesserten sich die Score-Punkte in beiden Gruppen gleichläufig, wobei Gruppe I einen ScoreWert von 11, Gruppe II einen von 9 jeweils durchschnittlich aufwies. Die Einteilung nach dem Schweregrad der Behinderung zeigte somit in beiden Gruppen initial einen schlechten subjektiven Zustand, der sich bis zum Follow-up an die Grenze vom guten zum befriedigenden Ergebnis wandelte. Die statistische Aufbereitung dieser Ergebnisse zeigte für beide Gruppen im Vergleich keine signifikanten Unterschiede.

In beiden Gruppen erkennt man eine vergleichbare Schmerzintensität, die nach Mobilisation mäßiggradig ausgeprägt ist und bis zum Follow-up auf ein gut erträgliches Maß schrumpft (Tab.3). In Gruppe 1 wurde die Schmerzintensivität am 11. Tag nicht gemessen, da die Patienten nicht mehr unter stationärer Überwachung waren. Auch hier lässt sich zwischen den Gruppen keine signifikante Differenz evaluieren.

\section{Diskussion}

Die konservative Therapie wurde bis zu den 80er-Jahren des letzten Jahrhunderts als die Standardtherapie in der Frakturversorgung von Wirbelfrakturen betrachtet. Bei der konservativen Therapie stehen bereits seit den 30er-Jahren des letzten Jahrhunderts 2 unterschiedliche Konzepte nebeneinander. Magnus stellte dabei die funktionelle Therapie ohne jede Repositionsmaßnahme in den Vordergrund, was er mit der Beobachtung, dass die meisten Frakturen an der Wirbelsäule trotz diverser Repositionsund Retentionsmaßnahmen im Sinne eines sekundären Korrekturverlusts 

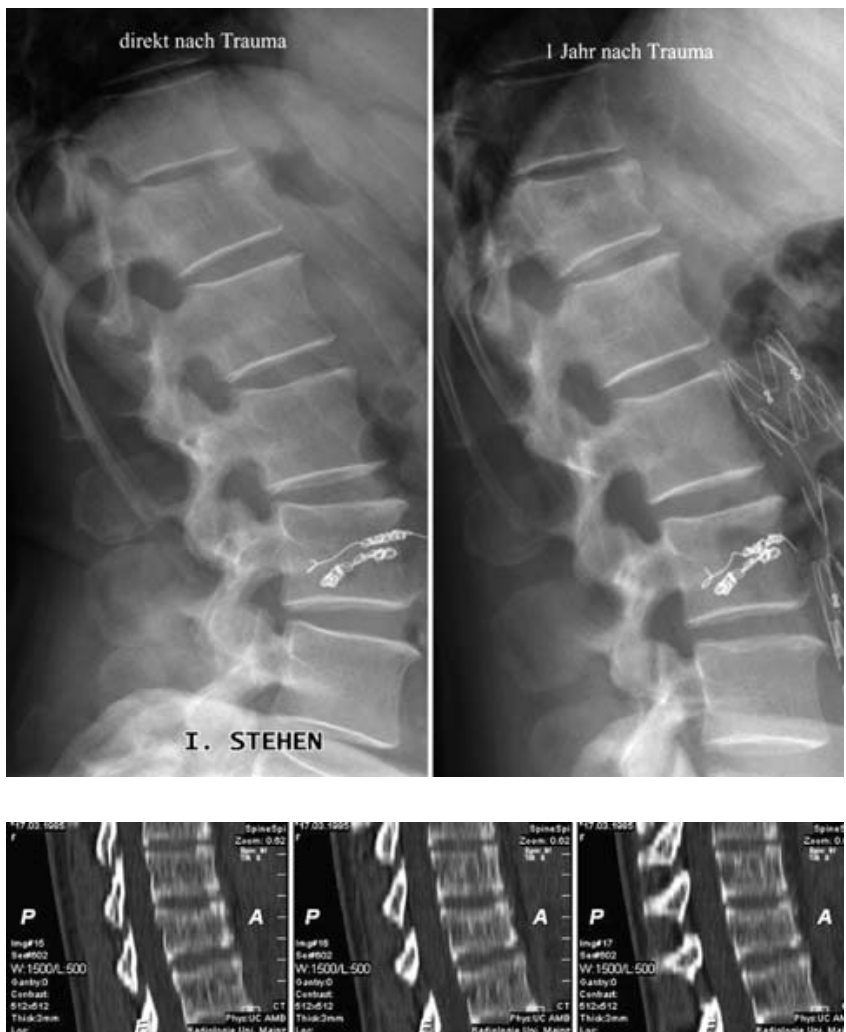

Abb. 6 22-j. Patientin T12-Fraktur (Gruppe 2), Unfall-CT und Veränderung im seitlichen Strahlengang 1 Jahr nach Trauma.
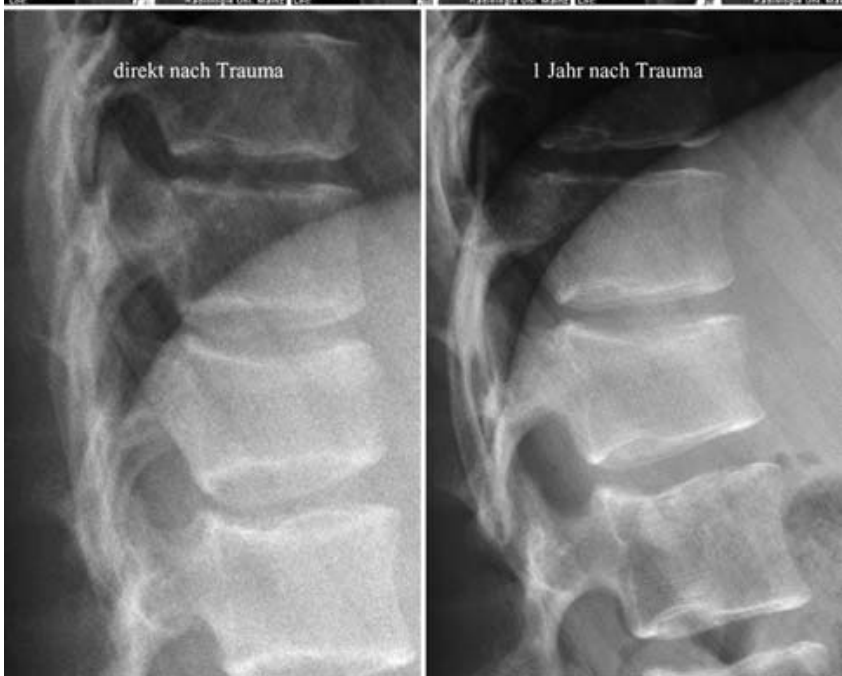

Tab. 3 Veränderungen der Werte in der visuellen Analogskala (VAS)

\begin{tabular}{lllll} 
& $\begin{array}{l}\text { VAS } \\
\text { posttrauma }\end{array}$ & $\begin{array}{l}\text { VAS } \\
\text { am 5. Tag }\end{array}$ & $\begin{array}{l}\text { VAS } \\
\text { am 11. Tag }\end{array}$ & $\begin{array}{l}\text { VAS } \\
\text { Follow-up }\end{array}$ \\
\hline Gruppe 1 & $\varnothing 7$ & $\varnothing 6$ nach Mobilisation & entfällt & $\varnothing 4$ \\
\hline Gruppe 2 & $\varnothing 9$ & $\varnothing 3$ Bettruhe & $\varnothing 6$ nach Mobilisation & $\varnothing 3$
\end{tabular}

nachsintern, begründete. Seine detaillierte Beschreibung der konservativen Behandlung mit initialer Immobilisation über wenige Tage und anschließender Mobilisation unter Abnahme der Schwere im Bewegungsbad mit folgender Mobilisation im Stützkorsett findet noch bis heute seine Anhänger [16]. Im Gegensatz zu Magnus postulierte Böhler
Abb. 5 57-j. Patient T12-Fraktur (Gruppe 1), Veränderung im seitlichen Strahlengang 1 Jahr nach Trauma. ten 2 Monaten die additive Immobilisation mit Bettruhe gefordert wurde. Im Bereich des thorakolumbalen Übergangs und der Lendenwirbelsäule propagierte er an vorderster Stelle die Reposition, welche stets bei wachem Patienten (zur Beurteilung des neurologischen Status) in der Regel im dorsalen Durchhang mit Lordorsierung durch Zug von oben durchgeführt werden sollte. Additiv postulierte er den Längszug an allen 4 Extremitäten. Nach Reposition sollte dann ein eng anmodellierter Gipsverband angelegt werden.

Ein gemeinsamer Aspekt der Therapiekonzepte von Böhler und Magnus ist die physiotherapeutisch geführte schmerzfreie Kräftigung der Rückenstrecker und der ventralen Rumpfmuskulatur, um ein sog. muskuläres Korsett zu bilden, welches ein dynamisches Gleichgewicht der kompromittierten Statik der Wirbelsäule herstellen soll.

Die konservative nicht operative Behandlung hat heute, insbesondere bei stabilen Frakturen, eine nach wie vor sehr große Bedeutung.

Dazu gehört unzweifelhaft die adäquate medikamentöse Behandlung (Analgetika, Antiphlogistika, Muskelrelaxanzien, neurotrope Substanzen, additiv auch das gesamte Spektrum der bei Osteoporose verabreichten Medikamente). Weiter hat sich bis heute die physikalische Therapie mit all ihren Facetten vor allem im deutschen Sprachraum etabliert. Ob und wann eher eine Ruhigstellung oder aber eine funktionelle kontrollierte Mobilisation erfolgen soll, wird nach wie vor kontrovers diskutiert. Die strenge Bettruhe bei konservativer Therapie wurde bereits 1928 als effizient postuliert [17]. Die Mieder- bzw. Korsettversorgungen etc. haben sich ebenfalls in der konservativen Therapie weitgehend durchgesetzt, obwohl die Datenlage hier nicht eindeutig ist.

Insbesondere bei Kompressionsfrakturen hat sich eine frühzeitige Mobilisation sinnvoller erwiesen als lang andauernde Bettruhe [18]. Der Vergleich des Therapieerfolgs verschiedener konservativer Methoden zeigt keine wesentlichen Unterschiede [19]. Auch in unserer eigenen Untersuchung, die die in Deutschland weitverbreiteten Methoden der frühfunktionellen Mobilisation mit und ohne initiale Immobilisation vergleichen, konnten wir keine signifikanten Unterschiede sowohl im klinischen als auch 
im radiologischen Outcome feststellen. Das klinische Endergebnis, gemessen mit dem Oswestry-Score, lag bei beiden Gruppen an der Grenze von allgemein gutem zu befriedigendem Ergebnis und entspricht den bisher publizierten Ergebnissen. Aus unseren Ergebnissen folgern wir, dass, bezüglich des Outcomes, durch eine initiale Immobilisation über eine knappe Woche keine besseren Ergebnisse zu erwarten sind.

Die konservative Therapie erweist sich als besonders geeignet bei Berstungsfrakturen ohne neurologische Funktionseinschränkung unter Berücksichtigung der Stabilitätskriterien. Ein Zusammenhang zwischen morphologischen Veränderungen und Beschwerden der Patienten konnte bisher nie sicher festgestellt werden. Dagegen spielt der Versicherungsstatus eine Rolle, denn einige Arbeiten zeigen, dass bei nicht versicherten Patienten mit Wirbelsäulenverletzungen bessere klinische Ergebnisse gefunden wurden. Die im Alter zunehmenden schmerzhaften Spondylarthrosen sind schwer von posttraumatischen Beschwerden zu unterscheiden. Nachteile der konservativen Therapie liegen in der schlechteren anatomischen Repositionsmöglichkeit und der längeren Immobilisation, die Risiken einer Operation werden hingegen vermieden.

Eine operative Behandlung entwickelte sich aber erst langsam in den letzten 200 Jahren. Erst Mitte des letzten Jahrhunderts wurden operative Verfahren populärer, was an der zunehmenden stetigen Technisierung und Verbesserung der bildgebenden Verfahren begleitet von Neuerungen, sowie der Entwicklung von stabilisierenden Implantaten lag. Dieser Prozess dauert bis heute und stellt ein unbeschreibliches Wachstum in der Wirbelsäulenchirurgie dar. Es ist zu erwarten, dass sich dieser Prozess immer weiter fortsetzt. Entscheidender Vorteil der operativen Verfahren, vor allem bei instabilen Situationen, gegenüber der konservativen Therapie, auch bei Patienten ohne neurologisches Defizit, ist die Möglichkeit der Frühmobilisation. Die durchschnittliche Dauer der Erstbehandlung im Krankenhaus lässt sich deutlich reduzieren, was neben einem Gewinn an Lebensqualität auch zu einer Erleichterung der Resozialisierung führt. Die Indikation zur operativen Versorgung von thorakolumbalen Wirbelsäulenfrakturen, insbesondere von Berstungsfrakturen bei erhaltenem posterioren Ligamentkomplex, wird nach wie vor kontrovers diskutiert, da konservative Maßnahmen ähnlich gute Ergebnisse erwarten lassen. Die Meinung, dass alle Berstungsbrüche operationswürdig sind, ist nicht zu halten. Auch erst ab einer Spinalkanalstenose bei fehlender Neurologie von über $50 \%$ eine Indikation zur operativen Intervention zu sehen, kann heute nicht vertreten werden. Differenzierter sind Betrachtungen, die das Risiko für neurologische Ausfälle als Begründung einer operativen Therapie verstehen. So wird eine Operationsindikation bei einer Spinalkanalstenose $\geq 35 \%$ in Höhe Th11$12, \geq 45 \%$ in Höhe $\mathrm{L} 1, \geq 55 \%$ in Höhe L24 postuliert [20].

\section{Schlussfolgerung}

Das Therapieziel der Behandlung thorakolumbaler Wirbelfrakturen ist für die operative und für die konservative Therapie das Gleiche. Form, schmerzfreie Funktion und Belastungsfähigkeit sollen erhalten bleiben bei regulärem Neurostatus. Sekundärziele sind frühzeitige Mobilisation, Pflegeerleichterung und Verhinderung von Komplikationen.

Bei entsprechender Indikation bietet die konservative Behandlung auch heute noch eine sinnvolle Alternative zur operativen Versorgung. In der Regel werden die aus den Klassifikationen bekannten Kriterien der Stabilitätsbeurteilung herangezogen.

Eine konservative Therapie ist heute vor allem bei stabilen Frakturen ohne neurologisches Defizit gerechtfertigt. Ist es nicht möglich, die o.g. Therapieziele im Rahmen der konservativen Therapie zu erreichen, ergibt sich heute die Indikation zum operativen Vorgehen.

\section{Literatur}

${ }^{1}$ MacKenzie EJ, Siegel JH, Shapiro S et al. Functional recovery and medical cost of trauma: an analysis of type and severity of injury. J Trauma 1988; 28: 281-297

2 Beck E. Konservative Behandlung von Frakturen und Luxationen von Thorax und Lendenwirbelsäule. Hefte zur Unfallheilkunde 1980; 149: 119-128

${ }^{3}$ Böhler L. Die Technik der Knochenbruchbehandlung. Wien: Maudrich; 1951: 318-480

${ }^{4}$ Magnus G. Zur Behandlung der Wirbelbrüche. Arch Klin Chir 1938; 191: 547-553

${ }^{5}$ Denis $F$. The three column spine and its significance in the classification of acute thoracolumbar spinal injuries. Spine 1983; 8: 817831

${ }^{6}$ McCormack T, Karaikovic E, Gaines RW. The load sharing classification of spine fractures. Spine 1994; 19: 1741-1744

7 Magerl F, Aebi M, Gertzbein SD et al. A comprehensive classification of thoracic and lumbar injuries. Eur Spine J 1994; 3: 184-201
${ }^{8}$ White AA, Panjabi MM. Clinical biomechanics of the spine. 2nd ed. Philadelphia: JB Lippincott Company; 1990: 53-125

${ }^{9}$ Holdsworth FW. Fractures, dislocations, and fracture-dislocations of the spine. J Bone Joint Surg [Br] 1963; 45: 6-20

10 Gercek E, Hartmann F, Kuhn S et al. Dynamic angular three-dimensional measurement of multisegmental thoracolumbar motion in vivo. Spine 2008; 33: 2326-2333

${ }^{11}$ Blauth M. Grundlagen der Wirbelsäulentraumatologie. In: Tscherne $\mathrm{H}$, Blauth $\mathrm{M}$, eds. Tscherne Unfallchirurgie - Wirbelsäule. Berlin, Heidelberg, New York: Springer; 1998: 1-59

${ }^{12}$ McAfee PC, Yuan HA, Fredrickson BE et al. The value of computed tomography in thoracolumbar fractures. An analysis of one hundred consecutive cases and a new classification. J Bone Joint Surg [Am] 1983; 65: 461-473

13 Magerl F, Engelhardt P. 3. Brust- und Lendenwirbelsäule - Verlaufsformen. In: Witt AN, Rettig H, Schlegel KF, eds. Orthopädie in Praxis und Klinik, Spezielle Orthopädie (Wirbelsäule - Thorax - Becken). Stuttgart, New York: Thieme; 1994: 3.82-3.132

${ }^{14}$ Blauth M, Knop C, Bastian L. Brust- und Lendenwirbelsäule. In: Tscherne $\mathrm{H}$, Blauth $\mathrm{M}$, eds. Tscherne Unfallchirurgie - Wirbelsäule. Berlin, Heidelberg, New York: Springer: 1998: 241-372

${ }^{15}$ Blauth M, Bastian L, Knop C et al. Interobserverreliabilität bei der Klassifikation von thorakolumbalen Wirbelsäulenverletzungen. Orthopäde 1999; 28: 662-681

${ }^{16}$ Magnus G. Die Behandlung und Begutachtung des Wirbelbruches. Arch Orth Unfallchir 1931; 29: 277-284

17 Schanz A. Praktische Orthopädie. Berlin: Springer; 1928

18 Solheim K. Spätergebnisse nach funktioneller Wirbelbruchbehandlung. Hefte zur Unfallheilkunde 1971; 108: 99-104

19 Werner B, Wehling H, Matthaes P. Vergleichende Nachuntersuchungsergebnisse aufgerichteter und konservativ behandelter Wirbelfrakturen der Brust- und Lendenwirbelsäule. Bruns' Beitrag zur klinischen Chirurgie 1972; 219: 735-743

${ }^{20}$ Hashimoto T, Kaneda K, Abumi K. Relationship between traumatic spinal canal stenosis and neurologic deficits in thoracolumbar burst fractures. Spine 1988; 13: 1268-1272

\section{Dr. med. Erol Gercek \\ Oberarzt}

Dr. med. Holger Meinig

Assistenzarzt

Dr. med. Frank Hartmann

Assistenzarzt

Dr. med. Eric Hanke

Oberarzt

Dr. med. Sven Oliver Dietz

Facharzt

Univ.-Prof. Dr. med. Dr. h. c.

Pol Maria Rommens

Leiter des Zentrums

Zentrum für Unfallchirurgie und

Orthopädie

Universitätsmedizin der Johannes-

Gutenberg-Universität Mainz

Langenbeckstraße 1

55131 Mainz

egercek@uni-mainz.de 